\title{
Computational Investigation of Shear Driven Mechanophore Activation at Interfaces
}

\author{
Meenakshi Sundaram, Meredith Silberstein ${ }^{1}$ \\ Sibley School of Mechanical and Aerospace Engineering, Cornell University
}

\begin{abstract}
Self-healing and self-reporting interfaces can alleviate damage related costs for both coatings and composites. In this paper we propose to utilize molecular units termed mechanophores, that undergo productive chemical transformations in response to force, to augment interfaces with self-healing and self-reporting functionality. We investigate the critical parameters for mechanophore-functionalized interfaces by building two computational models: a kinematic model with rigid non-interacting walls forming the interface and a molecular dynamics model with substrates of Lennard-Jones particles. In both the models the mechanophore is idealized as a coarse grained two-bead system governed by a double-well potential that emulates a force directed chemical reaction. Subjecting the interface models to shear, our study reveals that mechanophores progressively activate as interfacial displacement increases starting with the mechanophores aligned along the direction of shear. Subsequently we find that activation happens only if the bonds attaching the mechanophores to the substrates are strong enough to transmit the force necessary to activate the mechanophore. When substrates forming the interface are adhesive, the transmission of shear is delayed, in turn delaying activation of mechanophores. Adhesion-based changes in the conformation of the interface alters the extent of mechanophore activation. These results are a first step towards designing mechanophore-enabled self-reporting and self-healing composites and coatings.
\end{abstract}

Keywords: Interface, Double-well, Molecular dynamics, Self-Reporting, Self-Healing, Mechanochemistry

\section{Introduction}

Solid interfaces are prevalent both within materials and structures. Some ubiquitous examples include nanoparticle polymer composites [1], fiber-reinforced composites [2], and polymer coatings $[3,4,5]$. Interfaces with self-reporting $[6,7,8]$ and autonomous self-healing $[9,10]$ capabilities help manage damage and lower life cycle costs. The self-reporting approach provides a localized indication of the extent of damage and the autonomous selfhealing approach provides localized repair at the site of damage mimicking biological systems. In this work we propose to augment interfaces with mechanochemical functionality

\footnotetext{
${ }^{1} \mathrm{~ms} 2682 @$ cornell.edu

Preprint submitted to Extreme Mechanics Letters
}

August 26, 2015 
by bonding force triggered chemical units (termed mechanophores) [11] at the interface of two substrates. Several mechanophores have been developed that are promising for selfreporting and self-healing, including spiropyran that changes color[12], bis(adamantyl)-1,2dioxetane that luminesce [13], gem-dichlorocyclopropanated indene that releases an acid [14], gem-dibromocyclopropane that triggers cross-link formation [15, 16] and olefin metathesis catalyst with two tightly bound N-heterocyclic carbene ligands triggers polymerization of monomers or forms cross-links [17].

This paper is focused on mechanophore triggering at a sparsely populated interface subjected to shear loading. This focus on triggering intentionally neglects the chemical details that would be associated with a particular self-healing or self-reporting reaction in order to ascertain general design guidelines. We develop both a kinematic model and molecular dynamics model with coarse grained mechanophores and representative substrates. These models are used to interrogate the percentage of mechanophores activated as a function of interfacial displacement for different degrees of interactions between the mechanophore and substrate. Furthermore we examine how the presence of ambient temperature and adhesive interfacial conditions affect activation.

\section{Methods}

To build a model that captures mechanophore activation due to interfacial shear, we begin with a representative mechanophore and its interactions with a substrate. The mechanophore is simplified to two coarse grained beads bonded together. This bond is captured as a double-well potential that models an isomerization reaction with bond length change. This 1D potential model assumes that the entire reaction can be mapped onto a single reaction coordinate and that the force directed chemical reaction takes this same reaction pathway [18]. To construct the potential two Morse potentials (Eq. 1) were stitched together with a Bezier cubic spline (Fig. 1). The Bezier cubic spline and the Morse potential values were chosen to obtain extremal energy values (Table 1) in the range of those from quantum mechanical calculations of the mechanophore spiropyran [12]. A mechanophore is termed open (activated) if the bond length is greater than the position of the second potential minima and is termed closed otherwise.

Each bead of the mechanophore is covalently bonded to a substrate. The covalent substrate attachment is modeled with a Morse potential that captures the anharmonicities of the bond away from equilibrium [19] and the dissociation of the bond [20] with a finite energy. The Morse potential $V^{\text {Morse }}$ as a function of bond length $r$ is

$$
V^{\text {Morse }}=D\left[e^{-2 \alpha\left(r-r_{0}\right)}-2 e^{-\alpha\left(r-r_{0}\right)}\right]
$$

where $r_{0}$ and $D$ are the bond length and the depth at the equilibrium point respectively and $\alpha$ is a length scale parameter that is inversely proportional to the half-width of the potential well. The half-width $w_{1 / 2}^{\text {Morse }}$ is defined as the distance from the equilibrium point $r_{0}$ at which 
the applied force $d V^{\text {Morse }} / d r$ is maximum $\left(F_{\max }^{\text {Morse }}\right)$. The maximum force and half-width are related to the Morse potential parameters by:

$$
\begin{aligned}
F_{\max }^{\text {Morse }} & =\frac{\alpha D}{2} \\
w_{1 / 2}^{\text {Morse }} & =\frac{\ln (2)}{\alpha}
\end{aligned}
$$

For the interpretation of results, we define a finite bond break off point $r_{\mathrm{break}}^{\text {Morse: }}$

$$
r_{\text {break }}^{\text {Morse }}=r_{0}+3 w_{1 / 2}^{\text {Morse }}
$$

A mechanophore is termed detached if one of the attachment bonds extend beyond $r_{\mathrm{break}}^{\text {Morse }}$. The ranges of attachment bond parameters used in this study are given in Table 1.

The substrates can interact with each other and with the mechanophore beads through nonbonded interactions. The nonbonded interactions are modeled with Lennard-Jones (LJ) potentials (Fig. 1) — in the molecular dynamics model we use LJ 12-6 potential for each particle, in the kinematic model we use LJ 9-3 [21] which is an integration of the LJ 12-6 over the substrate. These LJ potentials are given by:

$$
\begin{aligned}
V^{\mathrm{LJ} 12-6} & =4 \epsilon\left[\left(\frac{\sigma}{r}\right)^{12}-\left(\frac{\sigma}{r}\right)^{6}\right] \\
V^{\mathrm{LJ} 9-3} & =\frac{2}{3} \pi \epsilon n \sigma^{3}\left[\frac{2}{15}\left(\frac{\sigma}{z}\right)^{9}-\left(\frac{\sigma}{z}\right)^{3}\right]
\end{aligned}
$$

where $\epsilon$ is the depth of the potential, $\sigma$ is the length scale associated with the potential, $n$ is the number density of the substrate, and $r$ and $z$ are the distances from the substrate particle and the substrate plane respectively. These parameter values are listed in Table 1.

The kinematic model is a potential energy minimization based analysis developed here to study the effect of interfacial shear on mechanophore activation. We lump each substrate forming a side of the interface into a rigid wall. The walls are positioned $4.71 \AA$ apart and and a mechanophore is placed in a particular orientation between the substrates. Each mechanophore bead is bonded to a point on the closer wall at the equilibrium attachment bond length. The walls are tangentially displaced in 400 equally spaced steps until the maximum distance necessary to either activate or detach the mechanophore system. At each step the potential energy, comprised of bonded attachments, nonbonded interactions, and the mechanophore double-well bond, is minimized. A single result is comprised of an average over 128 uniformly distributed mechanophore orientations. The orientations are sampled within the range found by the geometric constraint that both mechanophore beads must be placed between the rigid substrate walls.

A molecular dynamics (MD) model is developed here to study the impact of discrete substrate particles and intersubstrate interaction at room temperature on mechanophore activation. Though computationally expensive the MD approach is robust and can capture effects such as adhesion, substrate elasticity, and thermal perturbations. The energy barriers 
Table. 1. The extremal values for the cubic Bezier of the double-well and the parameters for the Morse and the Lennard-Jones potentials.

Double-well cubic Bezier

\begin{tabular}{|l|c|c|c|}
\hline & Minima 1 & Minima 2 & Maxima \\
\hline Distance $(\AA)$ & 7.03 & 13.19 & 11.89 \\
\hline Potential $(\mathrm{kcal} / \mathrm{mol})$ & -55.37 & -55.30 & -1.69 \\
\hline
\end{tabular}

Morse potentials

\begin{tabular}{|l|c|c|c|}
\hline & \multicolumn{2}{|c|}{ Double-well } & Attachment \\
\hline$D(\mathrm{kcal} / \mathrm{mol})$ & 61.03 & 50.79 & {$[8,140]$} \\
\hline$\alpha\left(\AA^{-1}\right)$ & 0.17 & 0.36 & {$[0.2,1.4]$} \\
\hline$r_{0}(\AA)$ & 7.69 & 13.73 & 4.0 \\
\hline Range $(\AA)$ & $(0,4.67)$ & $(15.08, \infty)$ & - \\
\hline
\end{tabular}

Lennard-Jones potentials

\begin{tabular}{|l|c|c|c|}
\hline & Substrate-mechanophore & Intra-substrate & Inter-substrate \\
\hline$\epsilon(\mathrm{kcal} / \mathrm{mol})$ & 1.23 & 12.31 & 6.16 \\
\hline$\sigma(\AA)$ & 3.12 & 2.57 & 5.14 \\
\hline$n\left(\AA^{-3}\right)$ & 0.06 & 0.06 & - \\
\hline
\end{tabular}

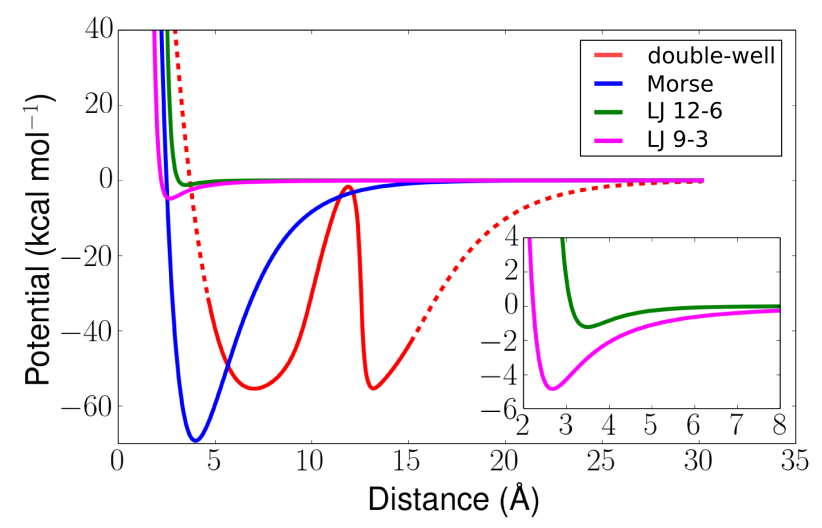

Fig. 1. Representative plot of potentials used in kinematic and MD simulations. The mechanophore double well potential is stitched from a cubic Bezier curve (-) flanked by two Morse potentials (- -). Inset: Zoomed in view of the nonbonded interactions near the well. Note that the LJ 9-3 potential is deeper than the corresponding LJ 12-6 potential. 


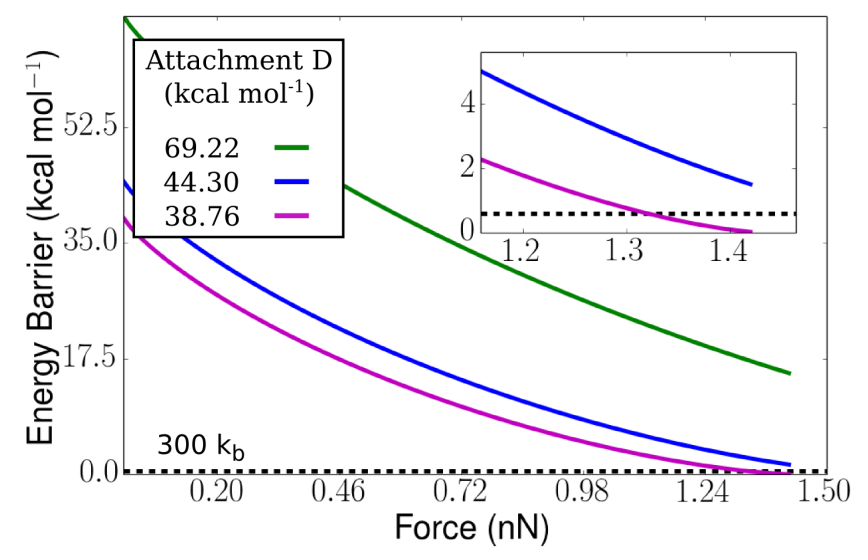

Fig. 2. Force modified energy barriers for dissociation of the substrate attachment bond for varying depths of the Morse potential with $\alpha=1.07 \AA^{-1}$. Inset: Zoomed-in view highlighting that the barrier is comparable to thermal energy for the forces close to $1.44 \mathrm{nN}$ that activates the mechanophore.

for the bonds are high relative to room temperature, but the force applied by the shear interface displacement performs work on the bonds and reduces the barrier to the regime for which thermal effects can be felt (Fig. 2).

Each substrate consists of Lennard-Jones particles arranged in a face centered cubic (FCC) lattice. The LJ parameters are set to gold [22] (Table 1). The simulation box is periodic in $\mathrm{x}$ and $\mathrm{y}$ and with a LJ 9-3 wall adjacent and parallel to one $\mathrm{x}-\mathrm{y}$ face emulating bulk and vacuum on the opposite face creating a free surface. The $\langle 111\rangle$ direction of the FCC crystal is aligned with the z-axis. Each substrate is $80 \AA$ wide in the $\mathrm{x}$ - and $\mathrm{y}$ - directions and $16 \AA$ deep in the z-direction. The substrate is step cooled from $1000 \mathrm{~K}$ to $300 \mathrm{~K}$ in 10 equally spaced temperature steps.

Two substrate configurations are assembled on top of each other with the exposed $\{111\}$ surfaces forming the interface as shown in Fig. 3b. Two LJ 9-3 walls that emulate bulk substrates flank the assembly. The substrates interact through a LJ 12-6 potential (Table 1) that was turned on and off to study effects of adhesion between the substrates. In the cases for which adhesion is turned on, the interface is equilibrated after the substrates are assembled but prior to the insertion of mechanophores (for the cases without adhesion this equilibration would be redundant since the substrates do not interact). For all cases, 8 mechanophores are inserted between the substrates at randomly selected orientations and locations within the criteria that both mechanophore beads are placed within the interface and that centre of mass of mechanophores are atleast $14 \AA$ apart. The mechanophore beads are bonded covalently to the nearest substrate particle. The whole interface is then equilibrated. Quasistatic shear is simulated by displacing a single atom thick layer on each end of the interface in opposite directions through 40 steps of $0.45 \AA$ each. At each step the interface is equilibrated (excluding the controlled displacement top and bottom atomic layers) and the mechanophore beads and the attached substrate particles are tracked. A single result is an average over 25 simulations. 


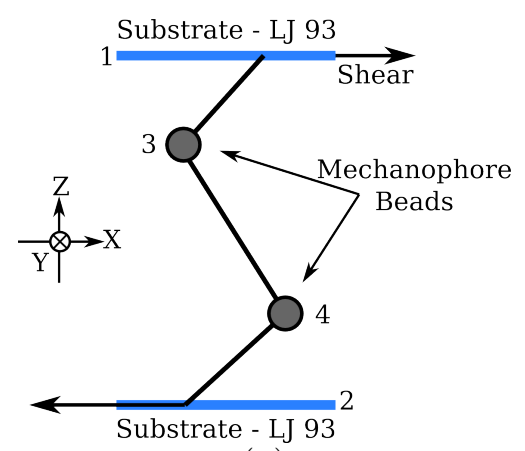

(a)

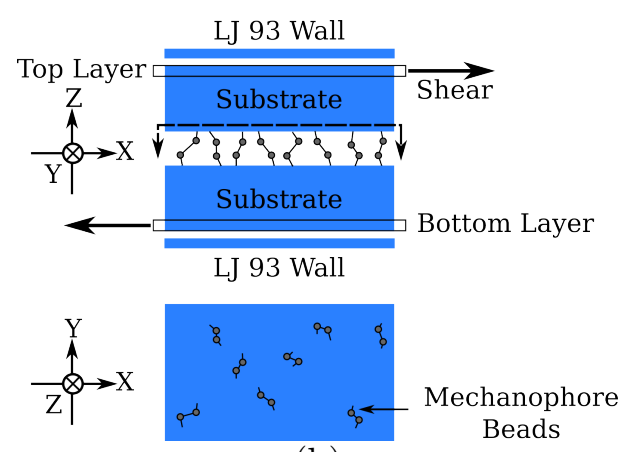

(b)

Fig. 3. Schematics of the two models for activation of mechanophores subjected to interfacial shear. (a) Kinematic model: Interface is formed by two LJ 9-3 walls with a randomly oriented coarse grained mechanophore in between. (b) MD model: Interface is formed by two atomistic substrates with eight randomly oriented and placed coarse grained mechanophores.

\section{Results and Discussion}

\subsection{Kinematic Model}

When mechanophores are rigidly attached to rigid substrates all mechanophores will progressively activate with interfacial displacement (Fig. 4a). Activation initiates at an interfacial distance close to the distance between the two minima of the double-well potential (slightly reduced due to the inclination of the mechanophore). Mechanophores that are oriented along the direction of shear activate first. To quantify mechanophore orientation we use the second order Legendre polynomial $\left(P_{2}\right)$ :

$$
P_{2}=\frac{3\left\langle\cos ^{2}(\theta)\right\rangle-1}{2}
$$

where $\theta$, is the angle with the direction of shear and $\langle>$ represents an average over a set of mechanophores. This function takes the value 1 for perfect alignment along the direction of shear, 0 for uniform random distributed orientations, and -0.5 for alignment perpendicular to the direction of shear. All mechanophores align themselves along the direction of shear given sufficient interfacial displacement, as indicated by the evolution of $P_{2}$ from 0.2 to 1 with interfacial displacement. The first mechanophores to open are those that are highly oriented along the shearing direction (Fig. $4 \mathrm{~b})$. Note that perfect alignment $\left(P_{2}=1\right)$ is not necessary for activation.

For non-rigid mechanophore to substrate attachments two kinds of behavior are observed. In Fig. 4a the progression of activation is plotted for three different bond potentials of the same $\alpha\left(\alpha=1.07 \AA^{-1}\right)$ and different $D$. When $D$ equals $69.22 \mathrm{kcal} \mathrm{mol}^{-1}$ (case 1 ) and $44.30 \mathrm{kcal} \mathrm{mol}^{-1}$ (case 2) activation coincides with the rigid attachment scenario with all mechanophores activating as the shear progresses. For $D$ equals $38.76 \mathrm{kcal} \mathrm{mol}^{-1}$ (case 3) no mechanophores activate with applied interfacial displacement. For case 3 all of the mechanophores detach from the substrate due to the weak substrate attachment (Fig. 4c), thereby removing the driving force for activation. 
To identify the range of parameters of the attachment potential that enable mechanophore activation we performed a grid search over $\alpha$ and $D$ - the parameters for the Morse potential, and evaluated what percent of mechanophores activated at each combination of parameters. As seen in Fig. 4d there are two distinct regimes - one for which $100 \%$ of the mechanophores activate and one for which $0 \%$ of mechanophores activate. The former regime occurs when the attachment potential is deep and narrow, while the latter regime occurs when the attachment potential is shallow and wide. The curve that separates the two regimes coincides with a rectangular hyperbola

$$
F_{\max }^{\mathrm{Morse}}=\frac{\alpha D}{2}=1.44 \mathrm{nN}=F_{\text {Activate }}^{\mathrm{Me}}
$$

where the left hand side is the maximum force that can be exerted by the Morse potential and the right hand side is the force required to activate the mechanophore (derived from the double-well potential). For this scenario with perfectly flat substrates and only bonded interactions, the mechanophore-substrate attachment bond must be capable of transmitting the force required to activate the mechanophores in order for any of the mechanphores to activate.

The presence of nonbonded interactions between the substrates and mechanophores perturbs the mechanophore response to interfacial shear (Fig. 5a). This perturbation is most apparent for case 2, which reaches only $3 \%$ activation when nonbonded interactions are present as compared to $100 \%$ activation with only bonded interactions. Revisiting the grid search now with nonbonded interactions present we notice a narrow band over which the transition from $2 \%$ activation to $98 \%$ activation occurs (Fig.5b). Some activation occurs for attachments with $F_{\max }^{\mathrm{Morse}}<F_{\text {Activate }}^{\mathrm{Me}}$ and some detachment occurs with $F_{\max }^{\mathrm{Morse}}>F_{\text {Activate }}^{\mathrm{Me}}$. To enable significant activation, the attachment potential needs to be reasonably stronger than the minimal requirement given by the force required to activate the mechanophore.

\subsection{Molecular Dynamics Model}

The presence of discrete substrate particles and ensemble dynamics as captured by the MD model does not significantly alter activation behavior (Fig. 5a). The substrate is effectively rigid as no substrate disintegration or shape change is observed during the MD simulation. Hence here too there are only two mechanisms at play, activation and detachment. There is a secondary thermal effect in case 2 for which activation reaches $5 \%$ with the total applied interfacial displacement instead of $3 \%$ predicted by the kinematic model.

Intersubstrate adhesion significantly influences mechanophore activation. Adhesion changes the configuration of the substrate atoms, resulting in substrates that are rougher and on average close together (Fig. 6a). In contrast to the previously studied scenarios, adhesion retards the interface displacement in relation to the prescribed shear boundary displacement (Fig. 6b). As most clearly evident in case 1, the reduced interface displacement reduces the mechanophore activation at a given boundary displacement. Furthermore, the more closely interacting substrates aids in both activation and detachment of the mechanophores as can be seen in the simulated cases. This adhesion study illustrates that atomistic details of the substrates play an important role in altering the progression of mechanophore activation. 


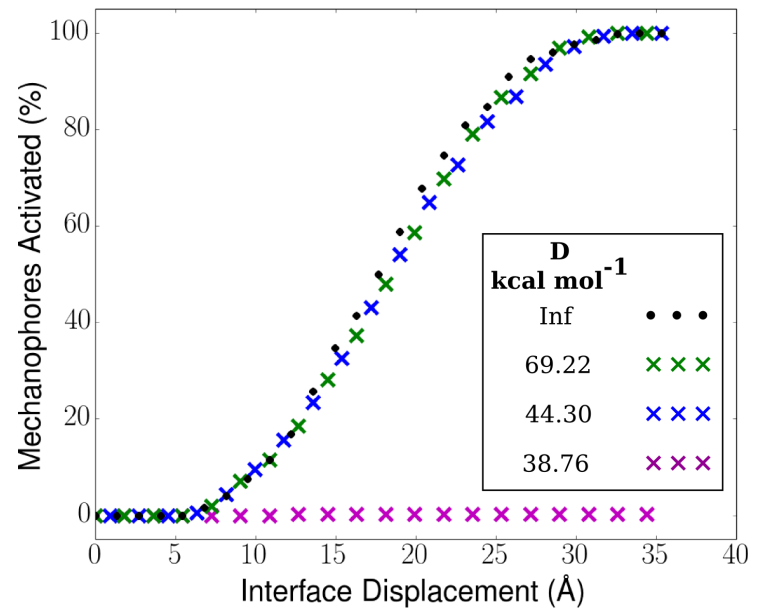

(a)

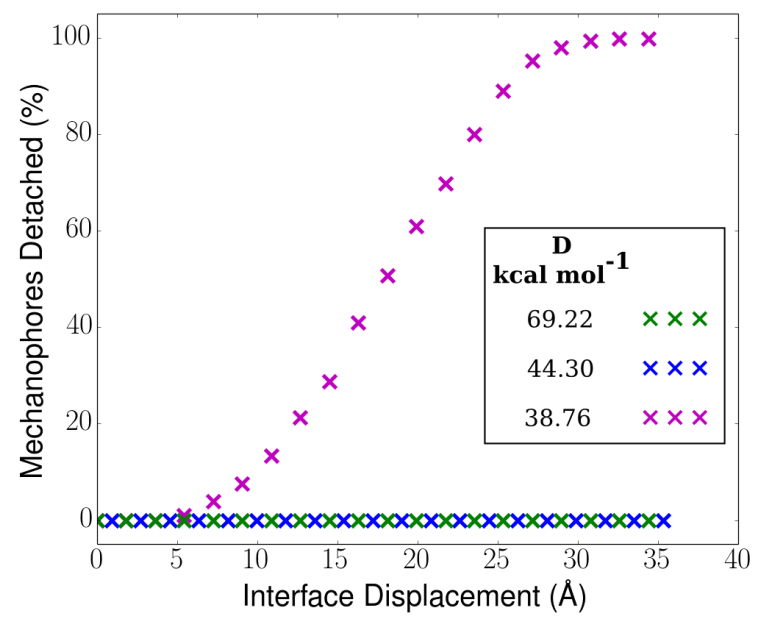

(c)

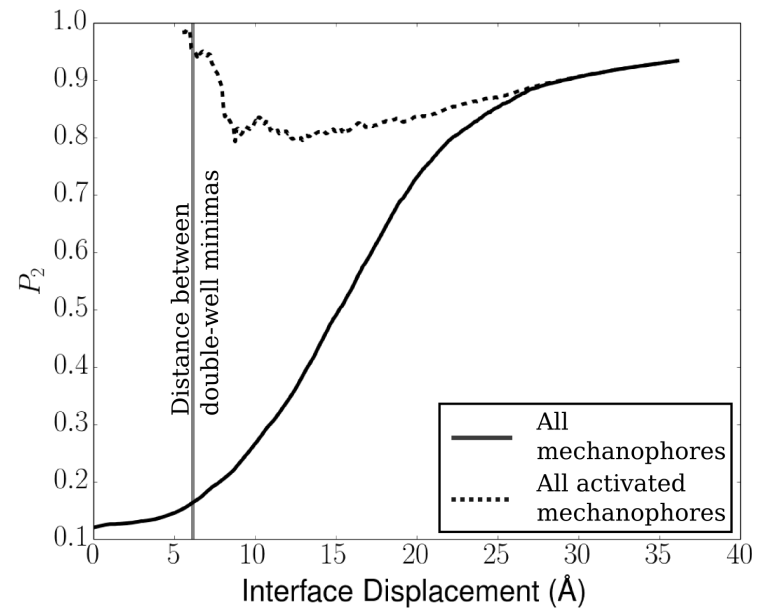

(b)

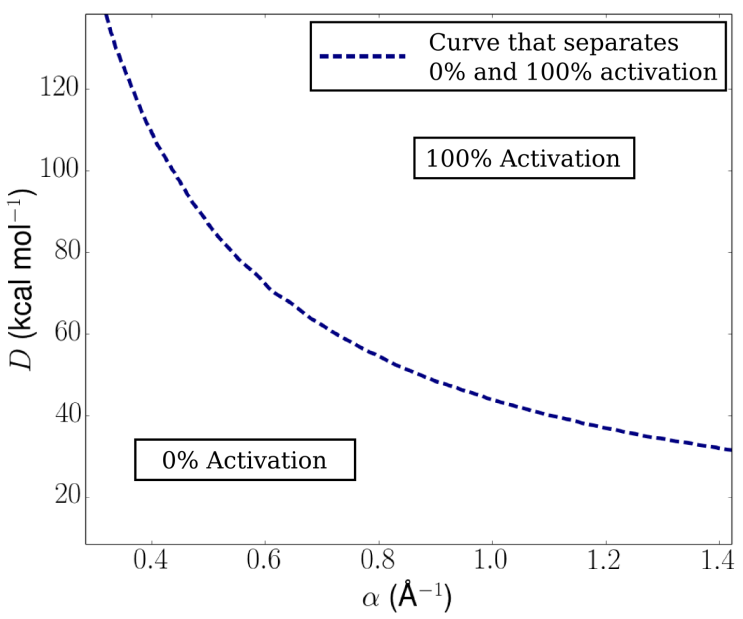

(d)

Fig. 4. Results for the kinematic model with only bonded interactions. (a) Activation trends of mechanophores with different attachment potential depths $D$ and $\alpha=1.07 \AA^{-1}$. Here "Inf" refers to a rigid attachment. (b) Orientation trends over all mechanophores and over the set of activated mechanophores when the attachments are rigid.(c) Dissociation trends of the mechanophores indicate that they complement the activation trends i.e., mechanophores either activate or dissociate. (d) The grid search over the space of the attachment potentials separates the space into two regimes - one where no mechanophores activate and one where all mechanophores activate is given sufficient interfacial displacement. 


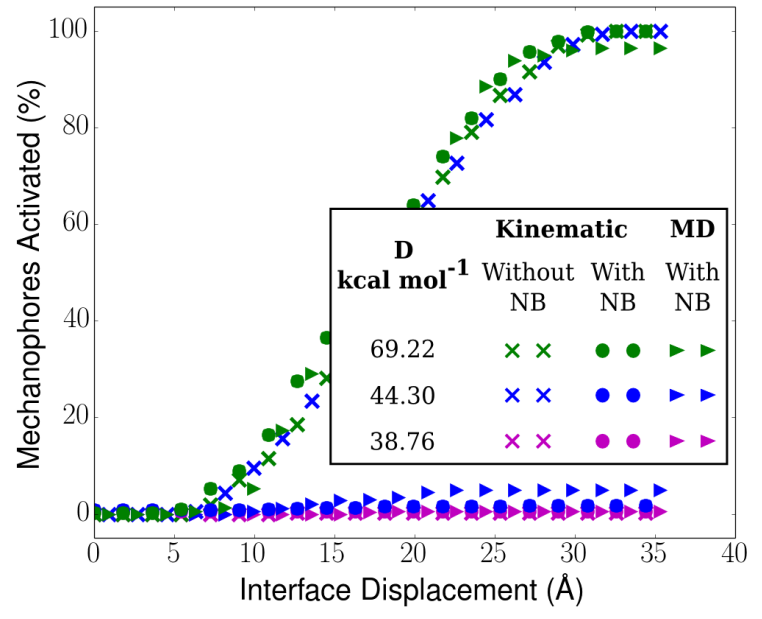

(a)

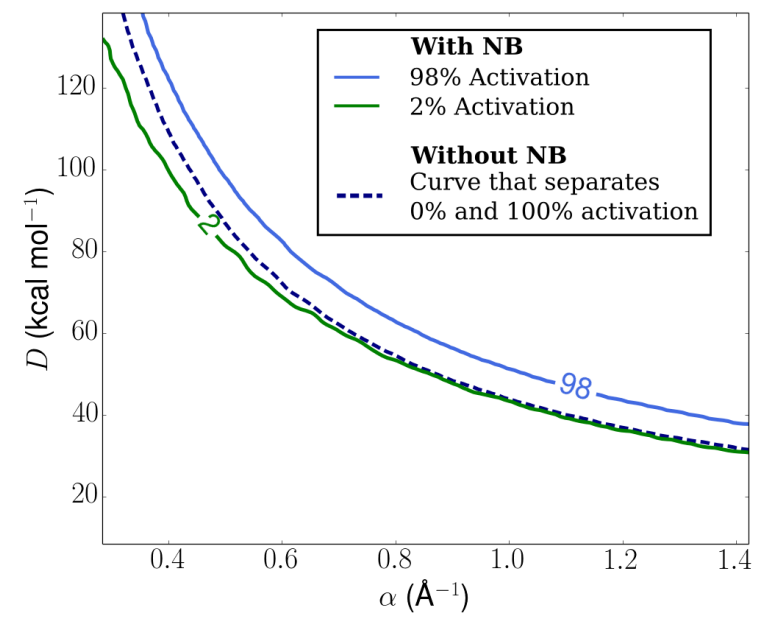

(b)

Fig. 5. Results of the kinematic model with and without nonbonded (NB) interactions, and of the corresponding MD model. (a) Activation trends with interfacial displacement. (b) In the presence of NB interactions the grid search performed with the kinematic model shows a narrow band over which the transition from no activation to complete activation happens.

\section{Conclusion}

We have constructed a kinematic model and a molecular dynamics model to simulate mechanophore activation at an interface subjected to shear. Our investigation reveals that mechanophore activation happens progressively with interfacial displacement starting with the mechanophores aligned along the direction of shear. The onset of activation happens when the interfacial distance nearly equals the distance between two equilibrium states of the mechanophore. If only limited interfacial displacement is expected in the composite or coating failure, then the distance between a potential mechanphore's equilibrium states may limit its utility. Our analysis highlighted that one critical aspect of interface design is to use mechanophore to substrate bonds that can survive forces larger than the mechanophore triggering force. However, some limited activation can occur even for weaker attachment bonds. Nonbonded interactions, temperature, and adhesion all contribute to both activation and detachment of mechanophores. If there is strong adhesion between the substrates, the conformation between the substrates change and the transmission of shear through the substrates is delayed, reducing the degree of activation for a given applied boundary displacement. This study offers insight for designing mechanophore functionalized self-healing and self-reporting interfaces for effective damage management. Based on these results, future work is needed to investigate the effect of specific substrates for prospective applications.

\section{Acknowledgement}

This material is based upon work supported by the National Science Foundation (NSF) under grant no. DMR 1307354 and used the Extreme Science and Engineering Discovery 

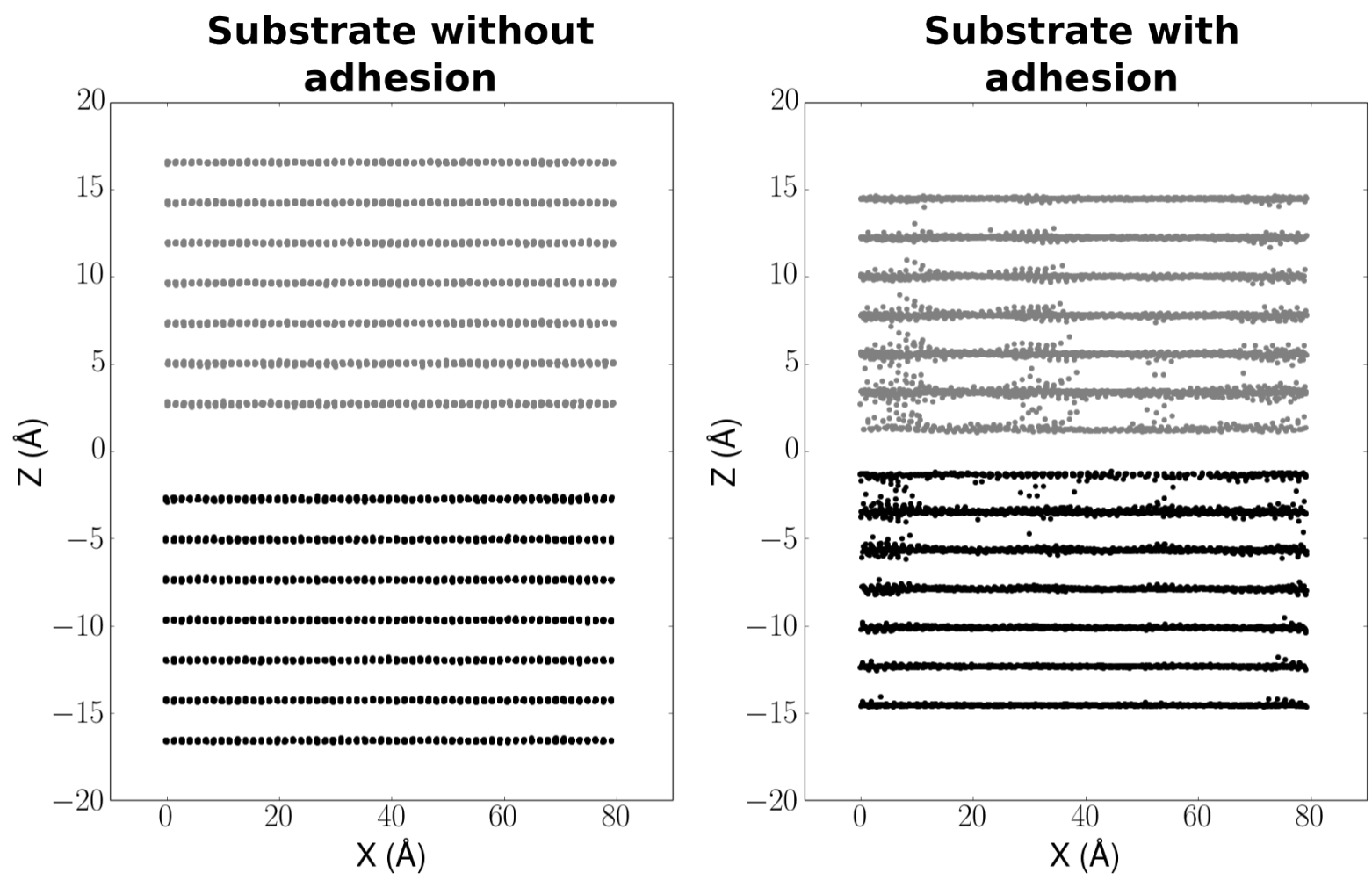

(a)

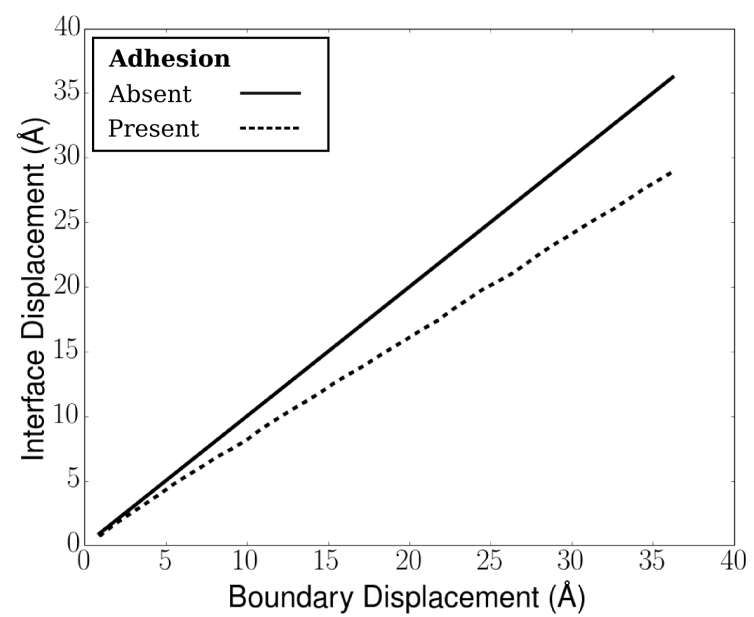

(b)

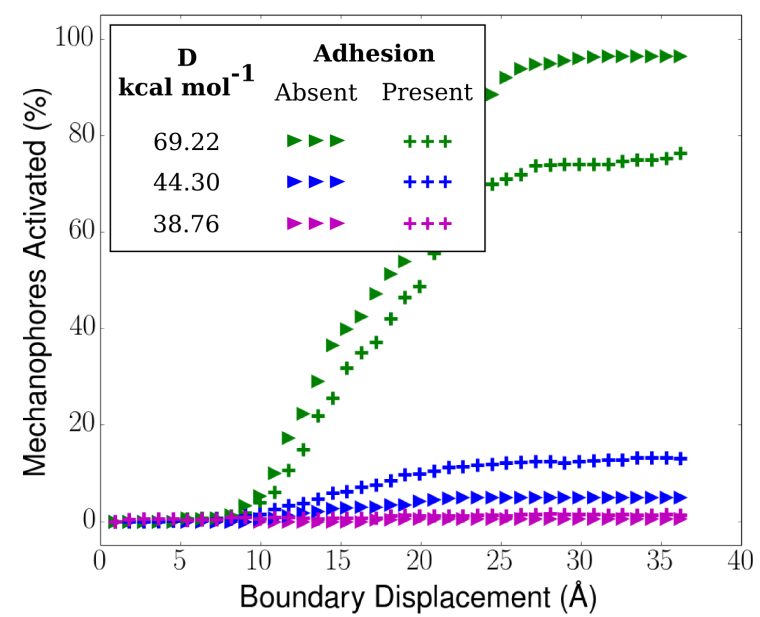

(c)

Fig. 6. Effect of inter-substrate adhesion on mechanophore activation determined by the MD model. (a) The interface configuration along the XZ plane is shown. The substrates come closer and they are rougher in comparison to the case without adhesion. (b) The interface displacement is less than the applied boundary displacement in the presence of adhesion. (c) Activation trends with and without adhesion. 
Environment (XSEDE), which is supported by National Science Foundation grant number ACI-1053575. We thank Dr. Jaewoo Kim of the Korea Institute of Science and Technology for useful discussions on this project.

\section{References}

[1] Anna C Balazs, Todd Emrick, and Thomas P Russell. Nanoparticle polymer composites: where two small worlds meet. Science, 314(5802):1107-1110, 2006.

[2] Pankar K Mallick. Fiber-reinforced composites: materials, manufacturing, and design. CRC press, Third edition, 2007.

[3] Haeshin Lee, Shara M Dellatore, William M Miller, and Phillip B Messersmith. Mussel-inspired surface chemistry for multifunctional coatings. Science, 318(5849):426-430, 2007.

[4] Indrani Banerjee, Ravindra C Pangule, and Ravi S Kane. Antifouling coatings: recent developments in the design of surfaces that prevent fouling by proteins, bacteria, and marine organisms. Advanced Materials, 23(6):690-718, 2011.

[5] Lei Zhai, Fevzi C Cebeci, Robert E Cohen, and Michael F Rubner. Stable superhydrophobic coatings from polyelectrolyte multilayers. Nano Letters, 4(7):1349-1353, 2004.

[6] Yan Hong and Ming Su. Multifunctional self-healing and self-reporting polymer composite with integrated conductive microwire networks. ACS Applied Materials \&3 Interfaces, 4(7):3759-3764, 2012.

[7] Gregory I Peterson, Michael B Larsen, Mark A Ganter, Duane W Storti, and Andrew J Boydston. 3d-printed mechanochromic materials. ACS Applied Materials \& Interfaces, 7(1):577-583, 2014.

[8] Xin Jin, Michael Götz, Sebastian Wille, Yogendra Kumar Mishra, Rainer Adelung, and Cordt Zollfrank. A novel concept for self-reporting materials: Stress sensitive photoluminescence in $\mathrm{ZnO}$ tetrapod filled elastomers. Advanced Materials, 25(9):1342-1347, 2013.

[9] Martin D Hager, Peter Greil, Christoph Leyens, Sybrand van der Zwaag, and Ulrich S Schubert. Selfhealing materials. Advanced Materials, 22(47):5424-5430, 2010.

[10] BJ Blaiszik, SLB Kramer, SC Olugebefola, Jeffrey S Moore, Nancy R Sottos, and Scott R White. Self-healing polymers and composites. Annual Review of Materials Research, 40:179-211, 2010.

[11] Charles R Hickenboth, Jeffrey S Moore, Scott R White, Nancy R Sottos, Jerome Baudry, and Scott R Wilson. Biasing reaction pathways with mechanical force. Nature, 446(7134):423-427, 2007.

[12] Douglas A Davis, Andrew Hamilton, Jinglei Yang, Lee D Cremar, Dara Van Gough, Stephanie L Potisek, Mitchell T Ong, Paul V Braun, Todd J Martínez, Scott R White, et al. Force-induced activation of covalent bonds in mechanoresponsive polymeric materials. Nature, 459(7243):68-72, 2009.

[13] Yulan Chen, AJH Spiering, S Karthikeyan, Gerrit WM Peters, EW Meijer, and Rint P Sijbesma. Mechanically induced chemiluminescence from polymers incorporating a 1, 2-dioxetane unit in the main chain. Nature Chemistry, 4(7):559-562, 2012.

[14] Charles E Diesendruck, Brian D Steinberg, Naoto Sugai, Meredith N Silberstein, Nancy R Sottos, Scott R White, Paul V Braun, and Jeffrey S Moore. Proton-coupled mechanochemical transduction: a mechanogenerated acid. Journal of the American Chemical Society, 134(30):12446-12449, 2012.

[15] Zachary S Kean and Stephen L Craig. Mechanochemical remodeling of synthetic polymers. Polymer, 53(5):1035-1048, 2012.

[16] Ashley L Black Ramirez, Zachary S Kean, Joshua A Orlicki, Mangesh Champhekar, Sarah M Elsakr, Wendy E Krause, and Stephen L Craig. Mechanochemical strengthening of a synthetic polymer in response to typically destructive shear forces. Nature Chemistry, 5(9):757-761, 2013.

[17] Robert TM Jakobs, Shuang Ma, and Rint P Sijbesma. Mechanocatalytic polymerization and crosslinking in a polymeric matrix. ACS Macro Letters, 2(7):613-616, 2013.

[18] Jordi Ribas-Arino and Dominik Marx. Covalent mechanochemistry: theoretical concepts and computational tools with applications to molecular nanomechanics. Chemical Reviews, 112(10):5412-5487, 2012.

[19] Stephen L Mayo, Barry D Olafson, and William A Goddard. Dreiding: a generic force field for molecular simulations. Journal of Physical Chemistry, 94(26):8897-8909, 1990. 
[20] Peter Hänggi, Peter Talkner, and Michal Borkovec. Reaction-rate theory: fifty years after Kramers. Reviews of Modern Physics, 62(2):251, 1990.

[21] JJ Magda, M Tirrell, and HT Davis. Molecular dynamics of narrow, liquid-filled pores. The Journal of Chemical Physics, 83(4):1888-1901, 1985.

[22] Ning Yu and Andreas A Polycarpou. Adhesive contact based on the Lennard-Jones potential: a correction to the value of the equilibrium distance as used in the potential. Journal of Colloid and Interface Science, 278(2):428-435, 2004. 